\title{
Vitamin B12 Inhibits Progression of Hypercholesterolemia and Atherosclerosis in Rabbits Fed a High Cholesterol Diet
}

$\underline{\text { Salim Abou-Khali1 }{ }^{*}, \text { M.D.C.M., Ron Poulsen }}{ }^{Y}$, Ph.D., Michael B. Stemerman ${ }^{\mathrm{a}}$, M.D., Sean Moore ${ }^{\mathrm{b}}$, M.B.B.Ch., F.R.C.P.(C), and Misbahuddin Zafar Alavi bc, Sc.D.

* Department of Otolaryngology, McGill University, Montreal, QC, Canada.

Ý Department of Biomedical Engineering and Pathology, McGill University, Montreal, QC, Canada.

a Department of Pathology, University of California at Riverside, CA, USA.

b Department of Pathology, McGill University, 3775 University Street, Montreal, QC, Canada H3A 2B4.

c To whom correspondence should be addressed.

\section{ABSTRACT}

The present study was undertaken to examine the effect of vitamin $B_{12}$ on serum cholesterol levels and development of aortic atherosclerosis in New Zealand White male rabbits fed a high cholesterol diet. Twenty rabbits were divided into 4 groups matched for their baseline serum cholesterol values. Groups 1 and 2 received a normal diet, whereas groups 3 and 4 had cholesterol mixed with their chow. After 5 weeks, the serum cholesterol of groups 3 and 4 increased by approximately 12 fold compared to groups 1 and 2 . At that point, vitamin $B_{12}$ was administered in the drinking water (1000 micrograms/l) to rabbits in groups 2 and 4 . At week 8 , the Vitamin $B_{12}$ dose was increased to 2400 micrograms/l for group 4. At 12 weeks into the study, rabbits in group 4 (high cholesterol diet plus vitamin $B_{12}$ ) had a serum cholesterol level of $919+/-270 \mathrm{mg} / \mathrm{dl}$ while those of group 3 (high cholesterol diet) had a serum cholesterol level of $1417+/-250 \mathrm{mg} / \mathrm{dl}$. At the end of the study, rabbit aortas were excised, fixed, and stained with fat-red-B, a dye for lipid. Comparison of the aortas of rabbits that received the lipid rich diet revealed that animals given additional vitamin $B_{12}$ had significantly decreased surface area of fat deposition. These data suggest the potential role for vitamin $B_{12}$ as a safe and inexpensive pharmacological intervention for the modification of the progression of human atherosclerosis.

\section{INTRODUCTION}

In the Western world, atherosclerosis is by far the most common cause of serious morbidity and death (1-5) and is responsible for the majority of cases of myocardial and cerebral infarction. Indeed, studies have shown that greater than half of total annual mortality in the United States is a consequence of the widespread 
complications of this disease (i.e., coronary artery disease, stroke, limb gangrene, etc.) (6) and similar statistics are reported in Canada (7). As such, atherosclerosis is a major health concern and identification of a safe, effective and inexpensive treatment should have widespread application.

Atherosclerosis affects mainly the medium and large elastic and muscular arteries; the aorta is severely affected and coronary artery atherosclerosis is associated with the serious complication of ischemic heart disease (2). The significant lesion is the fibrofatty plaque, which consists of a thickening arising within the intima and is typically confined to this region of the vessel (8). The plaque is composed of a core of lipids, mainly cholesterol, and a fibrous cap. With progression of the disease, the plaques increase in number and size, extending into the subjacent media and progressively obstructing the lumen of the artery, or when eroded or ruptured, serving as triggers for thrombosis, thereby compromising arterial blood flow (2-5). Despite extensive study, the atherogenic process has not yet been fully elucidated. It is probable that several environmental and intrinsic factors -- such as genes, growth factors, vessel wall cell types, and blood components -- interact to contribute to the complex atherogenic process (2-5).

There is a strong correlation between elevated serum cholesterol levels $(>200 \mathrm{mg} / \mathrm{dl})$ and the incidence of atherosclerosis $(9,10)$. Also, a high fat diet in humans is correlated with both coronary artery disease and high blood cholesterol levels (10). Most lipids, including cholesterol, are carried in the circulation bound to an apolipoprotein; together, this protein/lipid complex is termed a lipoprotein (11). The low density lipoprotein (LDL) carries the highest concentration of cholesterol and cells internalize LDL cholesterol for various functions (i.e., membrane maintenance, hormone synthesis) via the LDL receptor $(12,13)$. When cellular requirements for cholesterol are satisfied, a compensatory reduction in LDL mediated internalization occurs (12). Hence, either dysfunction of the LDL receptor (as in familial hypercholesterolemia) or excessive dietary cholesterol intake should increase blood cholesterol levels; this excess cholesterol likely makes a major contribution to atherosclerosis. In keeping with this hypothesis, most experimental animals fed a high cholesterol diet develop atherosclerosis (2-5,12-14).

Risk factors for atherosclerosis include age, male gender, family history, smoking, hypertension, and diabetes $(2-5,14)$. Some of these risk factors likely promote atherosclerosis via vascular endothelial cell damage $(2,4,10)$. In addition, recent studies have identified an elevated plasma homocysteine level as an important independent risk factor for atherosclerosis (15-21). Although endothelial injury is likely a contributing factor (22-24), the mechanism(s) by which homocysteine produces vascular damage are not precisely understood.

Homocysteine is formed during the metabolism of methionine, an essential sulfur-containing amino-acid (25). About $50 \%$ of the formed homocysteine enters a trans-sulfation pathway in which it is irreversibly combined with serine by a vitamin $\mathrm{B}_{6}$ dependent enzyme to form cystathionine. The remaining homocysteine is recycled into methionine by two distinct remethylation reactions, one of which is catalyzed by betainehomocysteine-methyltransferase. In the alternative reaction, of principal relevance to the present study, methionine synthase catalyses the remethylation of homocysteine using vitamin $\mathrm{B}_{12}$ (cyanocobalamine) and ethyltetrahydrofolate as cofactor and cosubstrate, respectively (25).

Studies have shown that markedly elevated homocysteine levels (which correlate with atherosclerosis) are associated with subjects with vitamin $\mathrm{B}_{12}$ deficiency, and an inverse correlation between serum $\mathrm{B}_{12}$ and homocysteine values has been reported by several investigators (25-32). Furthermore, studies have shown that vitamin $B_{12}$ supplement, in conjunction with other vitamin supplements (i.e., folate, vitamin $B_{6}$ ), can normalize blood homocysteine levels (25). Considering the potential involvement of vitamin $\mathrm{B}_{12}$ in protecting against atherosclerosis, the current experiment was designed to determine the protective effect of vitamin $\mathrm{B}_{12}$ treatment in an animal model of hypercholesterolemia-induced atherosclerosis. The data indicate that vitamin $\mathrm{B}_{12}$ treatment reduces high cholesterol diet-induced hypercholesterolemia and atherosclerosis and suggest that further studies should be undertaken to evaluate a potential role for vitamin $\mathrm{B}_{12}$ 
supplementation in the prevention of mortality and morbidity associated with human atherosclerosis.

\section{MATERIALS AND METHODS}

\section{Animals}

Twenty New Zealand White, age-matched, male rabbits (NZWR) (Charles River, St. Constant, Quebec, Canada) were used. After being acclimatized to the animal quarters for at least 2 weeks, the rabbits were divided into 4 groups matched for their baseline serum cholesterol values (Table 1). Each rabbit was housed separately with individual food and water supply.

\section{Hypercholesterolemia induction}

Rabbits in groups 1 and 2 were fed regular Purina chow. Rabbits of groups 3 and 4 were given the same chow mixed with cholesterol extracted from Lanolin (Fluka, Switzerland) and oleic acid (Fluka, Switzerland). The diet was prepared as previously described by Alavi et al. (33). Briefly, $7 \mathrm{~g}$ of cholesterol were dissolved in $100 \mathrm{ml}$ warm oleic acid and mixed with $70 \mathrm{~g}$ of normal rabbit chow.

\section{Determination of serum cholesterol}

Blood samples were collected from the marginal vein of the ear of each animal (from groups 1,2,3 and 4) at the onset of the study and at weeks $2,3,5,8$, and 12 . Samples were centrifuged and the serum concentration of cholesterol was determined using a modification of the enzymatic cholesterol measurement assay method of Allain et al. (34) (Diagnostics Cholesterol Reagent; Sigma, St. Louis, MO, USA). A simple main effect Ftest with Tukey's Honest Significant Different Test was used to evaluate serum cholesterol levels between the groups.

\section{Vitamin $\mathbf{B}_{12}$}

Water was provided to the rabbits in a closed bottle with a nipple device. At the end of week 5 of the study, vitamin $\mathrm{B}_{12}$ (Jamieson) was added to the drinking water of groups 2 and 4, initially at a concentration of 1000 micrograms/l. After 3 weeks of vitamin $B_{12}$ treatment, the concentration of vitamin $B_{12}$ in group 4 was increased to 2400 micrograms/1 for the remainder of the study.

\section{Assessment of atherosclerosis}

Twelve weeks following the initiation of the study, all animals were sacrificed by lethal administration of sodium pentobarbital. Aortas were excised and fixed with $4 \%$ formalin, and then stained with fat-red-B (Gelman Science Inc., Michigan, USA). The stained aortas were then photographed, the photos scanned into a computer and the surface areas of red-stained (fatty) and unstained white (no fat deposit) regions were quantified using a software program developed by the Pathology Department, McGill University. A t-test was used to make a statistical comparison between group 3 and group 4 .

\section{RESULTS}

\section{Effect of a high cholesterol diet on serum cholesterol}

As depicted in Figure 1 and Table 2 , the serum cholesterol level of control animals remained steady throughout the study at approximately $55 \mathrm{mg} / \mathrm{dl}$, such that serum cholesterol was not significantly different between group 1 (normal diet) and group 2 (normal diet plus vitamin $\mathrm{B}_{12}$ ) at any week. However, the cholesterol supplement (groups 3 and 4) raised the serum cholesterol level of rabbits approximately 12 fold at 
the fifth week of the study. Accordingly, the groups fed a high cholesterol diet were significantly different than the normal diet group (week $3,5,8$ and $12, p<0.01$ ).

\section{Effect of vitamin $\mathbf{B}_{12}$ supplementation on serum cholesterol}

From week 5 to week 8, the drinking water of group 2 (normal diet plus vitamin $\mathrm{B}_{12}$ ) and group 4 (high cholesterol diet plus vitamin $\mathrm{B}_{12}$ ) was supplemented with 1000 micrograms/l vitamin $\mathrm{B}_{12}$. In this period, the mean serum cholesterol concentration of group 3 (high cholesterol diet without vitamin $\mathrm{B}_{12}$ ) increased from $661+/-256$ to $989+/-330 \mathrm{mg} / \mathrm{dl}$. Also from week 5 to 8 , the serum cholesterol of group 4 (high cholesterol diet plus vitamin $\mathrm{B}_{12}$ ) increased from $622+/-270$ to $873+/-208 \mathrm{mg} / \mathrm{dl}$ ( Figure 1, Table 2). Since the difference between groups 3 and 4 did not reach statistical significance at week 8 , and considering that vitamin $B_{12}$ treatment induced a definite trend towards reducing serum cholesterol, the dose of vitamin $B_{12}$ was increased to 2400 micrograms/l from week 8 to week 12 . At the end of the study (week 12), the serum cholesterol concentration of rabbits receiving the cholesterol supplement without vitamin $\mathrm{B}_{12}$ treatment (group 3) was found to be $1417+/-250 \mathrm{mg} / \mathrm{dl}$ (Figure 1, Table 2). This concentration was more than twice the concentration measured at the end of week 5. Rabbits receiving vitamin $\mathrm{B}_{12}$ in addition to a cholesterol supplemented diet (group 4) had a much lower level of serum cholesterol at the end of week $12(919+/-197$ $\mathrm{mg} / \mathrm{dl}$ ) (Figure 1, Table 2), despite the fact both groups received the same amount of cholesterol in their diets. This difference between groups 3 and 4 was significant at week $12(p<0.01)$.

\section{Effect of vitamin $B_{12}$ supplementation on atherosclerosis}

As depicted in Figure 2, the total combined surface area of the red-stained (lipid containing) parts of the aorta of the rabbits fed a high cholesterol diet averaged $26.29+/-1.77 \mathrm{~A}^{2}$ units per animal whereas that of the rabbits fed the same atherogenic diet supplemented with vitamin $\mathrm{B}_{12}$ averaged $22.5+/-1.25 \mathrm{~A}^{2}$ units. This difference was statistically significant $(p<0.001)$.

\section{DISCUSSION}

In this study, vitamin $\mathrm{B}_{12}$ was demonstrated to significantly reduce hypercholesterolemia and the development of atherosclerosis in rabbits fed a high cholesterol diet. Other reports have examined the potential link between low plasma levels of vitamin $\mathrm{B}_{12}$ and risk factors for atherosclerosis. Notably, human studies have found that plasma homocysteine levels are higher at low plasma levels of vitamin $\mathrm{B}_{12}$ (25-32). However, the current study is the first to directly measure the impact of vitamin $\mathrm{B}_{12}$ on serum cholesterol levels and atherosclerosis in an experimental model of diet induced hypercholesterolemia and atherosclerosis. Considering the putative importance of hypercholesterolemia in the etiology of atherosclerosis, the present findings are of considerable interest.

The mechanism by which vitamin $\mathrm{B}_{12}$ reduced the magnitude of the serum cholesterol increase associated with the cholesterol supplemented diet is unclear. However, it is not likely related to the capacity of vitamin $\mathrm{B}_{12}$ to facilitate remethylation of homocysteine into methionine (25), since no correlation between elevated blood homocysteine and cholesterol has been shown (35-36). In the current study, it is most probable that vitamin $\mathrm{B}_{12}$ exerted its effect via the exogenous pathway of cholesterol metabolism and not by reducing endogenous production of cholesterol, as the activity of 3-hydroxy-3-methylglutaryl-CoA (HMGCoA) reductase (which catalyses the rate limiting reaction in endogenous cholesterol production) (11) is likely blocked by the hyperlipidemic diet. It is possible that vitamin $\mathrm{B}_{12}$ facilitated the transformation of cholesterol into other metabolites, or enhanced its elimination. The mechanism of this effect should be the focus of future studies. 
In the current study, the vitamin $\mathrm{B}_{12}$ supplement significantly impaired atherosclerotic progression. It is tempting to speculate that it may have done so via the reduction of plasma homocysteine, as high plasma levels of this amino acid in humans are associated with both atherosclerosis and low levels of plasma vitamin $\mathrm{B}_{12}$ (25-32). Furthermore, it is well established that vitamin $\mathrm{B}_{12}$ can reduce homocysteine levels through the methylation pathway, where it is a cofactor for methionine synthase and thus facilitates the remethylation of homocysteine into methionine (25). However, it is unclear the extent to which plasma homocysteine contributes to atherosclerosis progression in the current animal model, in which a high cholesterol diet is employed to induce atherosclerosis in an otherwise normal population of rabbits. Definitive confirmation of the contribution (or non-contribution) of homocysteine in the anti-atherogenic effect of vitamin $\mathrm{B}_{12}$ treatment in this model awaits further study.

Considering that the atherosclerosis was induced by a high cholesterol diet, and that vitamin $\mathrm{B}_{12}$ reduced the magnitude of serum cholesterol elevation caused by this diet, it is logical to attribute the attenuated progression of atherosclerosis to cholesterol clearance facilitated by vitamin $\mathrm{B}_{12}$. Indeed, this hypothesis is supported by the near perfect correlation between total serum cholesterol levels over the 12 weeks of the study and end stage (week 12) aortic atherosclerosis. Specifically, the high cholesterol fed rabbits supplemented with vitamin $\mathrm{B}_{12}$ had a total mean serum cholesterol level (weeks 1 to 12) which was $85.6 \%$ of the non-vitamin $\mathrm{B}_{12}$ supplemented high cholesterol fed rabbits (Table 2). The majority of this difference is accounted for during the last 4 weeks of the study corresponding to the increased dose of vitamin $B_{12}(2400$ micrograms/l in the drinking water). Most interestingly, the total aortic atherosclerosis build up in the $\mathrm{B}_{12}$ supplemented animals was $84.5 \%$ of control. Thus, there was a virtually one-to-one correlation between total mean serum cholesterol (a value representative of vasculature exposure to cholesterol) and final aortic atherosclerosis levels. Hence, this correlation supports the hypothesis that the lower aortic atherosclerosis associated with vitamin $\mathrm{B}_{12}$ treatment is secondary to vitamin $\mathrm{B}_{12}$ attenuation of induced hypercholesterolemia.

It was only during the last 4 weeks of the study, when the vitamin $B_{12}$ concentration was increased to 2400 micrograms/l in the drinking water, that the difference in serum cholesterol between groups 3 and 4 was significant $(\mathrm{p}<0.01)$, with group 4 (high cholesterol diet with vitamin $\mathrm{B}_{12}$ supplement) having a week 12 serum cholesterol concentration which was $35 \%$ less than the corresponding value measured in group 3

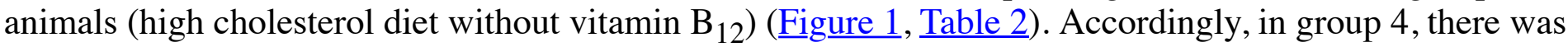
only a superficial, non-significant increase in serum cholesterol from week 8 to 12 (5.26\%) corresponding to the increased concentration of vitamin $\mathrm{B}_{12}$ supplement (Figure 1, Table 2). The robust capacity of vitamin $\mathrm{B}_{12}$ to essentially arrest serum cholesterol increases induced by the diet supplemented with cholesterol is emphasized by the observation that the cholesterol fed animals without vitamin $\mathrm{B}_{12}$ supplement had a rather large (43\%) increase in serum cholesterol level during the same period (Figure 1, Table 2). Hence, these data suggest that, at this concentration of vitamin $B_{12}$ supplement ( 2400 micrograms/l in the drinking water), serum cholesterol levels are approaching a threshold level. As 1000 micrograms/l did not attenuate the serum cholesterol increases to the same degree as did 2400 micrograms/l, it would seem that a certain concentration of vitamin $B_{12}$ is required before a significant cholesterol lowering effect is observed. Alternatively, a threshold time to treatment response may also have contributed to this observation.

Clearly, further studies are warranted to substantiate the observed effects. The concentration and time dependent characteristics of vitamin $B_{12}$ treatment on atherosclerosis and hypercholesterolemia should be evaluated in this model, as well as the long-term effects of vitamin $B_{12}$ treatment. Furthermore, it will be interesting to establish the capacity of vitamin $\mathrm{B}_{12}$ treatment to impede hypercholesterolemia and atherosclerosis in a setting where it is administered at the initiation of the hypercholesterolemic diet. 
Vitamin $\mathrm{B}_{12}$ was administered following prolonged consumption (5 weeks) of the hyperlipidemic diet. At this point, compared to control animals consuming a normal diet, serum cholesterol was dramatically elevated and atherosclerosis had likely begun (igure 1, Table 2). Nevertheless, vitamin $\mathrm{B}_{12}$ was able to significantly prevent further increases in serum cholesterol level and was associated with less advanced atherosclerosis. As such, the design of the current study has particular significance to the clinical situation, where patients will most often have pre-existing atherosclerosis and elevated cholesterol when the physician is consulted.

This is the first reported study which demonstrates that vitamin $\mathrm{B}_{12}$ supplementation following the induction of hypercholesterolemia by cholesterol supplemented diet inhibits further increases in serum cholesterol level, albeit through a yet to be specified mechanism. Also, the severity of atherosclerosis (which is likely secondary to the reduced serum cholesterol) was found to be significantly reduced. In conclusion, these results suggest that vitamin $B_{12}$ supplementation may prove useful as an inexpensive and effective measure to inhibit the progression of human atherosclerosis. Further basic studies should be conducted to validate progressing to a clinical evaluation of this hypothesis.

\section{ACKNOWLEDGMENTS}

The friendly help and excellent assistance of Dr. He Wang and Ms. Ghada Abou-Khalil is gratefully acknowledged. The authors express a very special thanks to Mr. Daniel Auld for the statistical analyses of the serum cholesterol.

\section{REFERENCES}

1. Uemura K, Pisa, Z. Trends in cardiovascular diease mortality in industrialized countries since 1950 . World Health Statistics Quarterly 41(3-4):155-178; 1988.

2. Moore S. Blood vessels and lymphatics. In: Damjanov I, Linder J, eds. Anderson's Pathology, 10th edition. Missouri: Mosby Year Book, Inc.; 1996.

3. Moore S, Alavi MZ. Vessel wall matrix : An overview. In: Woodford, Davignon and Sniderman, eds. Atherosclerosis X. Amsterdam: Elsevier; 1995; 369-372.

4. Ross R. Cell biology of atherosclerosis. Annual Review of Physiology 57: 791-804; 1995.

5. Parasad C, Chandra R.K. Atherosclerosis : A paediatric problem? Nutrition Research 11:1-5; 1990.

6. Strong JP. The natural history of atherosclerosis in childhood. Annals of the New York Acadamy of Sciences 623:9-15; 1991.

7. Reeder BA, Dagenais GR, Johansen H, et al. Cardiovascular disease in Canada 1993. Heart and Stroke Foundation of Canada Report; 1993.

8. Stary HC, Blankenhorn, DH, Chandler AB, et al. A definition of the intima of human arteries and of its atherosclerosis-prone regions. Arteriosclerosis and Thrombosis 12(1): 120-134; 1992.

9. Getz GS. An overview of atherosclerosis: A look to the future. Toxicologic Pathology 18(4 pt 1): 623-635; 1990.

10. Bierman L. Atherosclerosis and Other Forms of Arteriosclerosis. In: Wilson JD, Braunwald E, Isselbacher KJ, et al., eds. Harrison's Principles of Internal Medicine, 12th edition. New York: McGraw-Hill Inc.; 1991: 992-1001. 
11. Mayes, PA. Cholesterol Synthesis, Transport and Excretion. In: Murray RK, Granner DK, Mayes PA, Rodwell VW, eds. Harper's Biochemistry, 23rd edition. Norwalk, Connecticut: Appleton and Lange; 1993: 266-277.

12. Goldstein JL, Brown MS. Familial hypercholesterolemia: A genetic regulatory defect in cholesterol metabolism. American Journal of Medicine 58(2): 147-150; 1975.

13. Brown MS, Goldstein JL. A receptor-mediated pathway for cholesterol homeostasis. Science 232 (4746): 34-47; 1986.

14. Hegele RA. The pathogenesis of atherosclerosis. Clinica Chimica Acta 246(1-2): 21-38; 1996.

15. Malinow MR. Hyperhomocyst(e)inemia. A common and easily reversible risk factor for occlusive atherosclerosis. Circulation 81(6): 2004-2006; 1990.

16. Clarke R, Daly L, Robinson K, et al. Hyperhomocysteinemia: an independant risk factor for vascular disease. New England Journal of Medicine 324(17): 1149-1155; 1991.

17. Ueland PM, Refsum H, Brattstrom L. Plasma homocysteine and cardiovascular disease. In : Francis BR, ed. Atherosclerotic cardiovascular disease, hemostasis, and endothelial function. New York: Marcel Dekker; 1992.

18. Kang SS, Wong PW, Malinow MR. Hyperhomocyst(e)inemia as a risk factor for occlusive vascular disease. Annual Review of Nutrition 12:279-298; 1992.

19. Genest J Jr, Malinow MR. Homocyst(e)ine and coronary artery disease. Current Opinion in Lipidology 3:295-299;1992.

20. Stampfer MJ, Malinow MR, Willet WC, et al. A prospective study of plasma homocyst(e)ine and risk of myocardial infarction in US physicians. JAMA 268(7): 877-881; 1992.

21. Fortin LJ, Genest J Jr. Measurement of homocyst(e)ine in the prediction of arteriosclerosis. Clinical Biochemistry 28(2): 155-162; 1995.

22. Harker LA, Ross R, Slichter SJ, et al. Homocysteine induced atherosclerosis: The role of endothelial cell injury and platelet response in its genesis. Journal of Clinical Investigation 58(3): 731-741; 1976.

23. Wall RT, Harlan JM, Harker LA, et al. Homocysteine-induced endothelial cell injury in vitro: a model for the study of vascular injury. Thrombosis Research 18(1-2):113-121; 1980.

24. Harker LA, Harlan JM, Ross R. Effect of sulfinpyrazone on homocysteine induced endothelial injury and arteriosclerosis in baboons. Circulation Research 53(6): 731-739; 1983.

25. Elian KM. Plasma homocysteine and vascular disease. McGill Journal of Medicine 2(2): 107-112; 1996.

26. Brattstrom L, Israelsson B, Lindgarde F, Hultberg, B. Higher total plasma homocysteine in vitamin $\mathrm{B}_{12}$ deficiency than in heterozygosity for homocystinuria due to cystathionine beta-synthase deficiency. Metabolism: Clinical and Experimental 37(2):175-178; 1988.

27. Jacobsen DW, Gatautis VJ, Green R, et al. Rapid HPLC determination of total homocysteine and other thiols in serum and plasma: sex differences and correlation with cobalamin and folate concentrations in healthy subjects. Clinical Chemistry 40(6):873-881; 1994. 
28. Selhub J, Jacques PF, Wilson, PW, et al. Vitamin status and intake as primary determinants of homocysteinemia in an elderly population. JAMA; 270(22): 2693-2698; 1993.

29. Pancharuniti N, Lewis CA, Sauberlich HE, et al. Plasma homocyst(e)ine, folate, and vitamin $B_{12}$ concentrations and risk for early-onset coronary artery disease. American Journal of Clinical Nutrition 59(4):940-948; 1994.

30. Ubbink JB, Vermaak WJH, van der Merwe A, et al. Vitamin $\mathrm{B}_{12}$, Vitamin $\mathrm{B}_{6}$, and folate nutritional status in men with hyperhomocysteinemia. American Journal of Clinical Nutrition 57(1):47-53; 1993.

31. Dalery K, Lussier-Cacan S, Selhub J, et al. Homocysteine and coronary artery disease in French Canadian subjects: relation with vitamins $\mathrm{B}_{12}, \mathrm{~B} 6$, pyridoxal phosphate and folate. American Journal of Cardiology 75 (16): 1107-1111; 1995.

32. Malinow MR. Plasma homocyst(e)ine and arterial occlusive diseases: A mini-review. Clinical Chemistry 41(1): 173-176; 1995.

33. Alavi MZ, Galis Z, Li ZH, Moore S. Dietary alterations of plasma lipoproteins influence their interactions with proteoglycan enriched extracts from neointima of normal and injured aorta of rabbit. Clinical and Investigative Medicine 14(5): 419-431; 1991.

34. Allain CC, Poon LS, Chan CS, et al. Enzymatic determination of total serum cholesterol. Clinical Chemistry 20(4): 470-475; 1974.

35. Murphy-Chutorian DR, Wexman MP, Grieco AJ, et al. Methionine intolerance: a possible risk factor for coronary artery disease. Journal of the American College of Cardiology 6(4):725-730; 1985.

36. Malinow MR, Kang SS, Taylor LM, et al. Prevalence of hyperhomocyst(e)inemia in patients with peripheral arterial occlusive disease. Circulation 79(6):1180-1188; 1989.

\section{BIOGRAPHY}

Salim Abou-Khalil received his M.D.C.M. from McGill University (Montreal, Quebec, Canada) in 1996 and is presently a first year otolaryngology resident at McGill University. His research into the effects of vitamin $\mathrm{B}_{12}$ on hypercholesterolemia and atherosclersis was conducted during his third year of medical school at the Department of Pathology, Faculty of Medicine, McGill University.

Copyright (C) 1997 by MJM 